\title{
Inventário Abrangente de Prosperidade: Evidências de Propriedades Psicométricas no Brasil
}

\author{
Helenides Mendonça ${ }^{1}$ \\ Pontifícia Universidade Católica de Goiás, Goiânia-GO, Brasil \\ Pedro P. Pires \\ Universidade Federal do Rio de Janeiro, Rio de Janeiro-RJ, Brasil \\ Luciana Pacheco, Ariana Fidelis \\ Pontifícia Universidade Católica de Goiás, Goiânia-GO, Brasil \\ Isabella de M. Ramos Caiado \\ Centro Universitário Barão de Mauá, Goiânia-GO, Brasil
}

RESUMO

O Inventário Abrangente de Prosperidade (IAP) constitui um instrumento que acessa o bem-estar, concebendo o construto numa perspectiva hedônica. Este estudo avaliou as propriedades psicométricas do IAP em 501 adultos brasileiros. A metodologia de análise consistiu em combinação entre análise fatorial confirmatória e análise de redes, incluindo também análise da confiabilidade e validade convergente. Os resultados confirmam a estrutura de 18 fatores de primeira ordem e a de sete fatores de segunda ordem original. $\mathrm{O}$ artigo também apresenta uma terceira estrutura com quatro fatores de segunda ordem $-\chi^{2} / g l=1,75, \mathrm{CFI}=0,92$, TLI $=0,91$, $\mathrm{RMSEA}=0,042$. Foram detectadas diferenças para o ajuste, considerando o sexo dos participantes, em que o modelo obtido tem melhor ajuste para homens. O IAP apresenta validade convergente ao se correlacionar positivamente com felicidade e florescimento no trabalho. Portanto, a versão brasileira do IAP apresenta evidências iniciais de validade para uso em adultos no Brasil.

Palavras-chave: prosperidade no trabalho; escala de felicidade; escala de florescimento no trabalho.

\section{ABSTRACT - Comprehensive Inventory of Thriving: Evidence of Psychometric Properties in Brazil}

The Comprehensive Inventory of Thriving (CIT) is an instrument that accesses well-being, conceiving the construct from a hedonic perspective. This study evaluated the psychometric properties of the IAP in 501 Brazilian adults. The framework of analysis consisted of a combination of confirmatory factor analysis and network analysis, including reliability analysis and convergent validity. The results confirm the structure of 18 first order factors and seven second order factors from the original research. The present study also presents a third structure with four second order factors $-\chi^{2} / g l=1.75, \mathrm{CFI}=.92, \mathrm{TLI}=.91$, RMSEA $=.042$. Differences were detected for the fit considering the gender of the participants, where the model obtained presented a better fit for men. The CIT has convergent validity, correlating positively with happiness and flourishing at work. Therefore, the Brazilian version of the CIT presents initial evidence of validity for use with adults in Brazil.

Keywords: thriving; happiness; flourishing at work; comprehensive inventory of thriving.

\section{RESUMEN - Inventario Integral de la Prosperidad: Evidencias de Propiedades Psicométricas en Brasil}

El Inventario Integral de la Prosperidad (IIP) constituye un instrumento que accede al bienestar, concibiendo el constructo en una perspectiva hedónica. Este estudio evaluó las propiedades psicométricas del IIP en 501 adultos brasileños. La metodología de análisis consistió en combinación entre el análisis factorial confirmatorio y el análisis de redes, incluyendo también el análisis de la confiabilidad y validez convergente. Los resultados confirman la estructura de dieciocho factores de primer orden y la de siete factores de segundo orden original. El artículo también presenta una tercera estructura con cuatro factores de segundo orden $-\chi^{2} / g l=1.75, \mathrm{CFI}=.92$, TLI $=.91$, RMSEA $=.042$. Se detectaron diferencias para el ajuste considerando el sexo de los participantes, donde el modelo obtenido tiene mejor ajuste para hombres. El IIP presenta validez convergente al correlacionarse positivamente con felicidad y desarrollo profesional. Por lo tanto, la versión brasileña del IIP presenta evidencias iniciales de validez para uso en adultos en Brasil.

Palabras clave: prosperidad en el trabajo; escala de la felicidad; desarrollo profesional.

O termo "prosperar" denota o estado de funcionamento positivo amplo, abrangendo os aspectos mental, físico e social (Su, Tay, \& Diener, 2014). Atualmente o termo tem sido utilizado em escalas por melhor enfatizar 
a definição de saúde psicológica positiva ou bem-estar psicológico. A prosperidade na vida não pode ser compreendida somente a partir de sentimentos de felicidade, ou sentimento de realização, apoio e recompensa nos relacionamentos, mas integrando todos esses aspectos (Duan, Fei, Zhao, \& Guo, 2018; Su et al., 2014; Tong \& Wang, 2017).

Os estudos sobre prosperidade na vida são influenciados pelo debate acerca das perspectivas hedônicas e eudaimônicas. A primeira delas é caracterizada pela busca do prazer e satisfação dos desejos, sendo operacionalizada pelas emoções, afetos, prazer, felicidade e satisfação com a vida. A perspectiva eudaimônica estabelece ser a felicidade humana obtida pela busca do crescimento pessoal, tendo como propósito o alcance do melhor de si, como uma vida plena, próspera, com sentido e significado (Diener, 1984, 1999; Deci \& Ryan, 2008; Huppert \& So, 2013; Joshanloo, Sirgy, \& Park, 2018; Ryff, 1989; Su et al., 2014; Wiese, Tay, Su, \& Diener, 2018). Os estudos sobre bem-estar, florescimento e prosperidade na vida têm sido concebidos como fenômenos multidimensionais que compreendem aspectos de ambas as concepções.

$\mathrm{Na}$ esteira desse debate, com o propósito de estudar o bem-estar numa perspectiva abrangente e propiciar uma visão holística do funcionamento positivo, $\mathrm{Su}$ et al., (2014) desenvolveram uma medida que oferece indicadores objetivos relacionados a uma vida próspera, englobando aspectos hedônicos e eudaimônicos do funcionamento humano. Essa medida foi desenvolvida com dois objetivos, a saber: desenvolver ampla classificação acerca do bem-estar psicológico como construto, representando uma visão integral do funcionamento positivo; e disponibilizar medida que possui poder preditivo em relação a importantes consequentes de saúde e que seja útil a pesquisadores e profissionais ligados a esse campo do conhecimento. Ao acessar o bem-estar de forma ampla, representando uma visão integral do funcionamento positivo, o Inventário Abrangente de Prosperidade (IAP) se constitui numa medida abrangente que cobre uma lacuna na literatura sobre bem-estar como um fenômeno psicológico global.

O modelo integral de florescimento e prosperidade (Seligman, 2018), assim como as teorias psicológicas sobre o desenvolvimento humano, serviram de base para $\mathrm{Su}$ et al. (2014) construírem esse modelo de prosperidade composto por dezoito fatores, que se organizam em sete dimensões, a saber: 1 . bem-estar subjetivo (SWB) na forma de alta satisfação com a vida, sentimentos positivos e evitação dos sentimentos negativos; 2. relações enriquecedoras, que engloba o respeito, pertencimento, senso de comunidade, apoio, confiança e ausência de solidão; 3. interesse e engajamento nas atividades diárias; 4. significado e propósito na vida; 5 . senso de domínio e realização, englobando habilidades, aprendizado, realização autoeficácia e autoestima; 6. sentimentos de controle e autonomia; 7. otimismo.
Cada uma das sete dimensões de segunda ordem identificadas está ancorada teoricamente na literatura científica, sendo que a primeira dimensão se baseia na conceituação de Diener (1984) sobre o bem-estar subjetivo. A segunda dimensão se apoia no enriquecimento das relações positivas, destacando a confiança, o apoio e a importância das conexões sociais positivas para a saúde psicológica das pessoas e para a conquista de uma vida próspera (Lucas, Dyrenforth, \& Diener, 2008). A terceira dimensão é o engajamento, algumas vezes é referido como fluxo (Csikszentmihalyi, 1996), que ocorre quando os indivíduos são totalmente absorvidos em suas atividades e experimentam sensações de excitação energizada.

Outro aspecto positivo da saúde psicológica é o sentimento de domínio e maestria, que se refere à quarta dimensão. Para os autores, essa dimensão parte do pressuposto de que todos os indivíduos são dotados de habilidades que pode oferecer a sensação de realização e domínio. A quinta dimensão diz respeito à necessidade de autonomia, que pode ser entendida como a crença de que se tem controle sobre a própria vida e isso é um importante indicador de saúde psicológica positiva (Ryan \& Deci, 2000). A crença numa vida de significado e propósito está estruturada como a sexta dimensão e é considerada como um aspecto central do bom funcionamento psicológico. Embora muitas vezes associado à felicidade, são conceitos distintos (McGregor \& Little, 1998). Por fim, mas não menos importante, a sétima dimensão se refere ao otimismo. Para os autores, embora o otimismo não seja uma experiência fenomenológica, como o bem- estar subjetivo ou a realização, confere uma mentalidade positiva para o futuro, além de que as pessoas otimistas são mais propensas a evitar problemas emocionais que as pessoas pessimistas porque elas se esforçam para superar estressores e regular as emoções de forma mais eficaz (Duan, Guan, \& Gan, 2016; Wrosch, Jobin, \& Scheier, 2016).

Apesar de a medida de prosperidade desenvolvida por Su et al. (2014) avaliar o bem-estar numa perspectiva integral, mais recentemente, os relatos de pesquisa disponíveis na literatura científica demonstram que a medida foi validada até o momento em poucas populações, a saber: USA, China, Alemanha, Austrália, Rússia, Espanha, Turquia, Índia, Singapura, Argentina e México (Wiese, Tay, Su, \& Diener, 2018). Este estudo, portanto, traz uma contribuição à literatura científica ao oferecer evidências de validade da medida em língua portuguesa, especificamente no Brasil.

Considerando os resultados obtidos nos estudos de validação do IAP desenvolvidos em outros países, levantou-se a hipótese de que se obteria o melhor ajuste do modelo aos dados para a estrutura de 18 fatores (H1). Para análise de validade convergente, foram feitas análises de correlações com felicidade e florescimento no trabalho. Estudos desenvolvidos por Diener, Sandvik, e Pavot (2009) demonstram que pessoas bem-sucedidas 
e prósperas, que frequentemente experimentam afetos positivos, tendem a ser mais felizes. Os autores observam que a intensidade e a constância das experiências positivas refletem o aumento da felicidade a longo prazo. Assim é que a felicidade pode ser considerada como forte indicador de bem-estar subjetivo e de prosperidade na vida (Diener, 2006; Lyubomirsky \& Lepper, 1999). Com base nesse pressuposto, levanta-se a hipótese de que os fatores positivos do IAP estão associados positivamente à vivência de felicidade $(\mathrm{H} 2)$.

A prosperidade na vida se constitui como um fenômeno que se aproxima do conceito de florescimento, que se refere à experiência de se sentir bem e, ao mesmo tempo, mostrar-se capaz de desenvolver o seu potencial, ter autonomia sobre sua vida pessoal e profissional (Diener, Harter, \& Arora, 2010; Duan \& Xie, 2016; Hildenbrand, Sacramento, \& Binnewies, 2018; Mendonça \& Ferreira, 2018; Tang, Duan, Wang, \& Liu, 2016). Estudos demonstram que tais perspectivas do funcionamento positivo impactam no bem-estar pessoal de forma direta e indireta (Chaaban, Irani, \& Khoury, 2015; Ryan \& Deci, 2000). Com base nesses estudos, foi levantada a hipótese de que o florescimento no trabalho está associado positivamente com prosperidade na vida $(\mathrm{H} 3)$.

Frente aos argumentos apresentados, o objetivo do presente artigo é apresentar as propriedades psicométricas da versão em português-brasileiro do Inventário Abrangente de Prosperidade. Procurar-se-á examinar sua estrutura fatorial de primeira e segunda ordem, assim como evidências relativas a sua validade convergente.

\section{Método}

\section{Participantes}

Participaram desse estudo 501 respondentes, totalizando para o sexo masculino $n=150(29,9 \%)$ e para o sexo feminino $n=351(70,1 \%)$, com idade acima de 18 anos, $(M=32,87 ; D P=11,63)$. O tempo médio de atividades de trabalho foi de 12,81 anos $(D P=10,75)$. A amostra é caracterizada por um nível de escolaridade elevado, sendo que 215 (40,00\%) participantes apresentam nível educacional até o nível secundário completo, 95 (19,00\%) com nível superior completo e 190 (38,00\%) com formação continuada em pós-graduação.

\section{Instrumentos}

Inventário Abrangente de Prosperidade (IAP; Su et al., 2014). Escala composta por 54 itens, estruturados em 18 fatores de primeira ordem, sendo três itens para cada fator, e organizados numa estrutura de ordem superior com sete dimensões. Os participantes responderam ao IAP em uma escala Likert de cinco pontos, variando de 1 "discordo totalmente" a 5 "concordo totalmente". São exemplos de itens de cada um dos fatores de primeira ordem: 1. Apoio - "Há pessoas das quais eu posso depender para que me ajudem", "Há pessoas que me dão apoio e incentivo, "Existem pessoas que gostam de mim"; 2. Comunidade - "Eu me disponho a ajudar quando minha comunidade precisa que algo seja feito", "Eu convido meus vizinhos para virem a minha casa", "Eu procuro formas de ajudar meus vizinhos quando eles precisam"; 3. Confiança - "Eu posso confiar nas pessoas em minha sociedade", "As pessoas em minha vizinhança são confiáveis", "A maioria das pessoas que eu conheço são honestas"; 4. Respeito - "As pessoas me respeitam", "As pessoas são educadas comigo", "Eu sou tratado com o mesmo respeito que as outras pessoas"; 5. Solidão - "Eu me sinto solitário", "Frequentemente me sinto excluído", "Não há ninguém com quem eu me sinta próximo"; 6. Pertencimento - "Eu sinto que pertenço a minha comunidade", "Eu sinto que pertenço ao meu estado", "Eu sinto que pertenço ao meu país"; 7. Engajamento - "Eu fico totalmente concentrado nas atividades que faço", "Na maioria das atividades que eu faço, me sinto energizado", "Eu fico entusiasmado quando trabalho em algo"; 8. Habilidades - "Eu uso as minhas habilidades diariamente na minha vida", "Eu frequentemente uso meus talentos", "Eu faço as coisas que eu sou bom todos os dias"; 9. Aprendizado - "Eu aprendi algo novo ontem", "Aprender coisas novas é importante para mim", "Eu sempre aprendo algo todos os dias"; 10. Realização - "Eu estou alcançando a maioria dos meus objetivos", "Eu estou realizando minhas ambições", "Eu estou no caminho de alcançar meu sonho"; 11. Autoeficácia - "Eu posso ser bem-sucedido se me dedicar a isso", "Eu tenho confiança de que posso lidar com eventos inesperados", "Eu acredito que sou capaz na maioria das coisas"; 12. Autoestima - "O que eu faço na vida tem valor e vale a pena", "As coisas que eu faço contribuem para a sociedade", "O trabalho que eu faço é importante para outras pessoas"; 13. Controle (todos os itens reversos) - "Outras pessoas tomam a maioria das decisões em minha vida (R)", "As escolhas que eu faço na vida não são realmente minhas (R)", "Outras pessoas decidem o que eu posso ou não fazer (R)"; 14. Sentido e propósito - "A minha vida tem um claro senso de propósito", "Eu tenho encontrado um sentido de vida satisfatório", "Eu sei o que dá sentido à minha vida"; 15. Otimismo - "Eu sou otimista sobre meu futuro", "Eu tenho um olhar positivo sobre a vida", "Eu espero mais coisas boas do que ruins na vida"; 16 . Satisfação com a vida - "Em vários aspectos, minha vida está próxima do meu ideal", "Eu estou satisfeito com minha vida", "A minha vida está indo bem"; 17. Sentimentos positivos "Eu me sinto positivo a maior parte do tempo", "Eu me sinto feliz a maior parte do tempo", "Eu me sinto bem a maior parte do tempo"; 18 . Sentimentos negativos (todos os itens reversos) - "Eu me sinto negativo a maior parte do tempo (R)", "Eu tenho sentimentos infelizes a maior parte do tempo (R)", "Eu me sinto mal a maior parte do tempo (R)". 
Escala de Florescimento no trabalho (Mendonça, Caetano, Ferreira, Sousa, \& Silva, 2014). Instrumento composto por oito itens que se estruturam em um único fator. Os itens são dispostos em uma escala Likert de sete pontos, sendo 1 "discordo completamente" a 7 "concordo completamente". A medida original obteve $\alpha=0,85$. São exemplos de itens: "Em meu trabalho, sou competente e capaz de fazer as atividades que são importantes para mim", "O meu trabalho contribui para que eu leve uma vida com propósito e significado".

Escala de Felicidade (Spagnoli, Caetano, \& Silva, 2010). Escala composta por quatro itens que se estruturam em um único fator. No estudo original, o coeficiente alfa variou de 0,73 (grupo de 30 a 34 anos) a 0,80 (grupo com idade entre 25 e 29 anos), com um valor médio de 0,77 para a amostra global. Neste estudo, a escala de felicidade ficou composta por três itens com coeficiente de fidedignidade de 0,76 . Os itens estão dispostos em uma escala Likert de sete pontos, sendo 1 "de modo nenhum" a 7 "inteiramente". Exemplo de item: "Algumas pessoas são geralmente muito felizes. Gozam a vida independentemente do que aconteça, tirando o máximo de tudo. Em que medida esta caracterização o(a) descreve?"

\section{Procedimentos}

Primeiramente, o projeto de pesquisa foi submetido e aprovado no Comitê de Ética em pesquisa da PUC Goiás. Na sequência, obteve-se a autorização da autora do Inventário Abrangente de Prosperidade (IAP), Rong Su (2014), para a validação da medida no contexto brasileiro. O inventário foi traduzido de acordo com as normas da Comissão Internacional de Testes (ITC, 2010) e os procedimentos sugeridos por Borsa, Damásio, e Bandeira (2012). A versão em inglês foi submetida a tradução para o português por dois tradutores bilíngues. Prosseguiu-se com o processo de síntese das versões traduzidas por um juiz, com o objetivo de se chegar a uma versão única do instrumento, mantendo a equivalência entre as versões traduzidas e o instrumento original, de modo a garantir as correspondências: semântica, idiomática, experiencial e conceitual. Foi realizado um estudo piloto para averiguar a adequação dos itens, chegando-se à conclusão de sua adequação ao contexto do Brasil.

Os instrumentos utilizados neste estudo foram aplicados de forma física e eletrônica. Em ambas as modalidades de aplicação, os voluntários que concordaram com a pesquisa assinaram o Termo de Consentimento Livre e Esclarecido (TCLE), seguindo-se a aplicação dos instrumentos conforme suas diretrizes técnicas. A coleta de dados foi realizada em campi universitários, organizações privadas e públicas, coordenadas pelos autores do estudo.

\section{Análise dos Dados}

Para a análise dos dados, foram empregados os softwares R v.3.5.3 (R Core Team, 2018), combinado ao R Studio v1.1.463 - incluindo os pacotes Psych (Revelle,
2018), EGAnet (Golino \& Chirstensen, 2019), Lavaan (Rosseel, 2018) e SemTools (Jorgensen, 2018). O procedimento de análise da estrutura do Inventário Abrangente de Prosperidade (IAP) foi o de análise fatorial confirmatória (AFC), que, segundo Brown (2015), pode ser considerado um modelo restritivo de análise fatorial, em que a relação entre itens e fatores podem ser especificados. $\mathrm{O}$ método fornece, além da análise das cargas fatoriais e matrizes, índices de ajuste que abordam a aproximação entre a matriz extraída das observações e a matriz estimada pelo modelo. Foi escolhido o estimador MLM - parâmetros de máxima verossimilhança estimados com erro padrão e $\chi^{2}$ ajustado à média, robusto à não normalidade da distribuição dos resultados.

Dentre os índices escolhidos para a análise de ajuste pode-se listar $\chi^{2}, \chi^{2} / g l$, Comparative Fit Index (CFI) robusto, Tucker-Lewis Fit Index (TLI) robusto e Root Mean-Square Error of Approximation (RMSEA) robusto, todos ajustados ao estimador MLM. Como indicadores de ajuste, o presente trabalho assume $\chi^{2}$ não significativo, $\chi^{2} / g l<3,00$, CFI $>0,90$, TLI $>0,90$ e RMSEA $<0,08$ (Hair, 2014). Considerando análise multigrupo, foram empregues cinco tipos disponibilizados pelo pacote SemTools. Para esse objetivo, foi estimado um: 1. modelo configural sem aplicar restrições, 2. igualdade para cargas fatoriais (invariância métrica), 3. interceptos (escalar), 4. médias estimadas para as variáveis latentes e, por fim, 5. os resíduos (invariância restrita) (Jorgense, 2018). Para avaliar os resultados, foram considerados o cálculo do $\Delta \chi^{2}, \Delta$ CFI robusto e $\triangle$ RMSEA robusto.

Ao longo das análises de dados, foram empregados também procedimentos de análise de redes com um objetivo exploratório, principalmente, para avaliar possíveis estruturas subjacentes para os fatores considerando uma estrutura de segunda ordem. Estruturas foram identificadas empregando o algoritmo de walktrap sugerido por Reichardt e Bornholdt (2006) e implementado no pacote EGAnet (Golino \& Christensen, 2019).

Após a análise dos itens a serem retidos, foram estimados os coeficientes de consistência interna para os fatores de primeira e segunda ordem. O coeficiente de consistência interna escolhido foi o ômega de McDonald, conforme as recomendações de Revelle e Zinbarg (2009). Apenas após esse último passo, foi executada análise de validade convergente, por modelagem de equações estruturais.

\section{Resultados}

Primeiro foram modelados apenas os fatores de primeira ordem, com o objetivo de confirmar as 18 dimensões principais representadas pelo Inventário Abrangente de Prosperidade (IAP). O processo inicial indicou ajuste de $\chi^{2}(g l=1224)=2046,36(p<0,001) ; \chi^{2} / g l=1,67 ; \mathrm{CFI}=0,93$; $\mathrm{TLI}=0,92 ; \quad \mathrm{RMSEA}=0,04 \quad(\mathrm{IC}=95 \% \quad[0,037 ; 0,043])$. Todos os itens apresentaram carga fatorial superior ao 
mínimo de 0,30. Em seguida, foram realizadas tentativas de ajustar o modelo considerando os fatores de segunda ordem, conforme sugerido pelos autores do instrumento. A solução resultou em depreciação do ajuste para $\chi^{2}(g l=1342)=2485,70(p<0,001) ; \chi^{2} / g l=1,85 ; \mathrm{CFI}=0,91$; TLI $=0,90 ; \quad$ RMSEA $=0,045 \quad(\mathrm{IC}=95 \% \quad[0,042 ; 0,048])$. Apesar da convergência, a estrutura original prevê fatores de alta ordem com apenas uma variável latente subordinada, o que representa redundância métrica.
A partir desse ponto, foi iniciado um processo exploratório com o objetivo de verificar outras estruturas possíveis com distribuição adequada das variáveis latentes. Foram extraídos os escores fatoriais com o objetivo de executar análises de rede utilizando o estimador glasso e configuração de tuning de 0,50 . A análise resultou no gráfico disponível na Figura 1, que contém bordas representativas das associações entre as dimensões de primeira ordem.

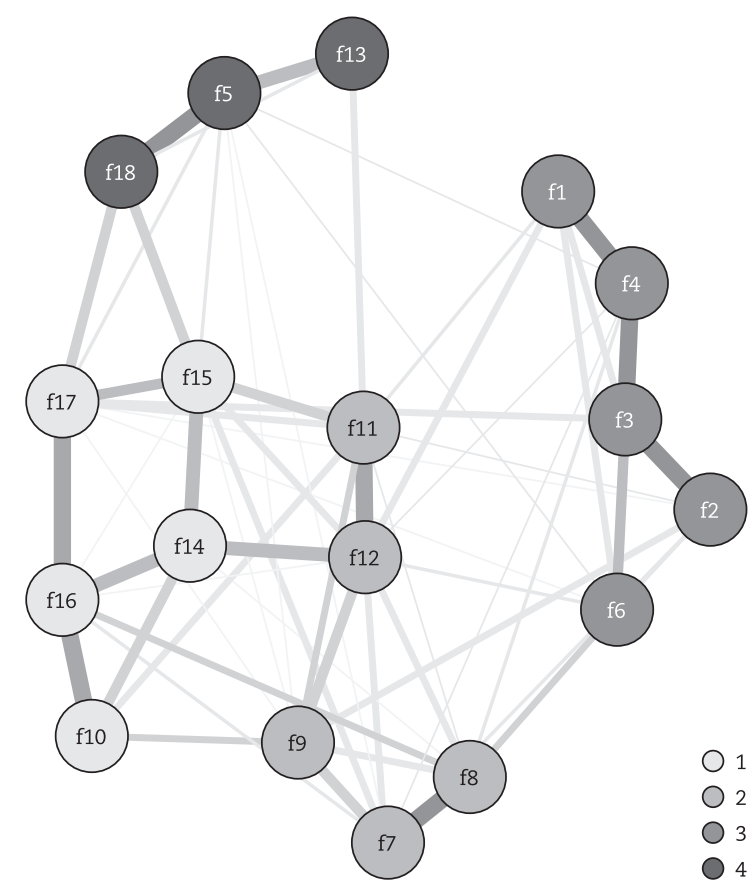

Figura 1. Rede de Relações entre Dimensões de Primeira Ordem

(Tons de Cinza Representam cada Comunidade Detectada)

Reichardt e Bornholdt (2006) abordam que comunidades em rede consistem em subgráficos que podem ser detectados. Os resultados indicam a existência de quatro comunidades: H1 - realização (F10), sentido (F14), otimismo (F15), satisfação (F16) e emoções positivas (F17); H2 - flow (F7), habilidades (F8), aprendizado (F9), eficácia (F11) e autoestima (F12); H3 - apoio (F1), comunidade (F2), confiança (F3), respeito (F4) e pertencimento (F6), e; H4 - solidão (F5), controle (F13) e emoções negativas (F18).

A partir das comunidades localizadas, foi executada uma nova AFC com o objetivo de testar os fatores de segunda ordem. A estrutura com os quatro fatores resultou em ajuste de $\chi^{2}(g l=1353)=2365,19(p<0,001)$; $\chi^{2} / g l=1,75 ; \quad$ CFI $=0,92 ; \quad$ TLI $=0,91 ; \quad$ RMSEA $=0,042$ $(\mathrm{IC}=95 \%[0,039 ; 0,045])$. Cargas fatoriais dos itens encontram-se na Tabela 1, não havendo cargas inferiores a 0,30 . Coeficientes dos caminhos de ordem superior estão disponíveis na Tabela 2.

Ao analisar comparativamente os três modelos obtidos por meio do comando ANOVA do Lavaan, o modelo apenas com os fatores de primeira ordem foi considerado o de melhor ajuste quando comparado ao modelo original $\left(\Delta \chi^{2}(\Delta g l=118)=426,3 ; p<0,001\right)$ e ao modelo novo proposto no presente artigo $\left(\Delta \chi^{2}(\Delta g l=129)=315,07\right.$; $p<0,001)$. O modelo original e o proposto não apresentam diferença estatística significativa em termos de ajuste $\left(\Delta \chi^{2}(\Delta g l=11)=152,27 ; p<0,001\right)$. 
Tabela 1

Cargas Fatoriais para o Modelo de Fator Geral, Quatro Fatores de Segunda Ordem e 18 Fatores de Primeira Ordem Incluindo Carga Fatorial, Erro Padrão (EP) e p-Valor

\begin{tabular}{|c|c|c|c|c|c|c|c|}
\hline Fator & carga fatorial & $E P$ & $p$-valor & Fator & carga fatorial & $E P$ & $p$-valor \\
\hline F1. Apoio & & & & F10. Real. & & & \\
\hline PAP1 & 0,35 & 0,05 & $<0,001$ & PRE1 & 0,90 & 0,04 & $<0,001$ \\
\hline PAP2 & 0,73 & 0,04 & $<0,001$ & PRE2 & 0,86 & 0,04 & $<0,001$ \\
\hline PAP3 & 0,71 & 0,04 & $<0,001$ & PRE3 & 0,79 & 0,04 & $<0,001$ \\
\hline F2. Comunidade & & & & F11. Eficácia & & & \\
\hline PCOM1 & 0,43 & 0,04 & $<0,001$ & PAEF1 & 0,52 & 0,04 & $<0,001$ \\
\hline PCOM2 & 0,73 & 0,06 & $<0,001$ & PAEF2 & 0,75 & 0,04 & $<0,001$ \\
\hline PCOM3 & 0,83 & 0,05 & $<0,001$ & PAEF3 & 0,81 & 0,04 & $<0,001$ \\
\hline F3. Confiança & & & & F12. Autoestima & & & \\
\hline PCONF1 & 0,73 & 0,04 & $<0,001$ & PAUE1 & 0,83 & 0,04 & $<0,001$ \\
\hline PCONF2 & 0,75 & 0,04 & $<0,001$ & PAUE2 & 0,76 & 0,03 & $<0,001$ \\
\hline PCONF3 & 0,65 & 0,04 & $<0,001$ & PAUE3 & 0,67 & 0,03 & $<0,001$ \\
\hline F4. Respeito & & & & F13.Controle & & & \\
\hline PRESP1 & 0,82 & 0,04 & $<0,001$ & PCONT1 & 0,78 & 0,05 & $<0,001$ \\
\hline PRESP2 & 0,77 & 0,04 & $<0,001$ & PCONT2 & 0,50 & 0,05 & $<0,001$ \\
\hline PRESP3 & 0,71 & 0,05 & $<0,001$ & PCONT3 & 0,82 & 0,05 & $<0,001$ \\
\hline F5. Solidão & & & & F14. Sentido & & & \\
\hline PSOL1 & 0,70 & 0,06 & $<0,001$ & PSEN1 & 0,73 & 0,03 & $<0,001$ \\
\hline PSOL2 & 0,76 & 0,06 & $<0,001$ & PSEN2 & 0,89 & 0,04 & $<0,001$ \\
\hline PSOL3 & 0,48 & 0,05 & $<0,001$ & PSEN3 & 0,76 & 0,04 & $<0,001$ \\
\hline F6. Pertencimento & & & & F15. Otimismo & & & \\
\hline PPER1 & 0,74 & 0,04 & $<0,001$ & POT1 & 0,84 & 0,03 & $<0,001$ \\
\hline PPER2 & 0,88 & 0,05 & $<0,001$ & POT2 & 0,90 & 0,04 & $<0,001$ \\
\hline PPER3 & 0,84 & 0,05 & $<0,001$ & POT3 & 0,76 & 0,03 & $<0,001$ \\
\hline F7. Flow & & & & F16. Satisfação & & & \\
\hline PENG1 & 0,63 & 0,04 & $<0,001$ & PSAT1 & 0,78 & 0,03 & $<0,001$ \\
\hline PENG2 & 0,80 & 0,04 & $<0,001$ & PSAT2 & 0,93 & 0,04 & $<0,001$ \\
\hline PENG3 & 0,71 & 0,03 & $<0,001$ & PSAT3 & 0,88 & 0,03 & $<0,001$ \\
\hline F8. Habilidades & & & & F17. Emoções Positivas & & & \\
\hline PHAB1 & 0,83 & 0,04 & $<0,001$ & PSPOS1 & 0,85 & 0,03 & $<0,001$ \\
\hline PHAB2 & 0,85 & 0,04 & $<0,001$ & PSPOS2 & 0,91 & 0,03 & $<0,001$ \\
\hline PHAB3 & 0,72 & 0,04 & $<0,001$ & PSPOS3 & 0,91 & 0,03 & $<0,001$ \\
\hline F9. Aprendizado & & & & F18. Emoções Negativas & & & \\
\hline PAPR1 & 0,72 & 0,06 & $<0,001$ & PSNE1 & 0,86 & 0,06 & $<0,001$ \\
\hline PAPR2 & 0,62 & 0,03 & $<0,001$ & PSNE2 & 0,92 & 0,07 & $<0,001$ \\
\hline PAPR3 & 0,79 & 0,05 & $<0,001$ & PSNE3 & 0,87 & 0,07 & $<0,001$ \\
\hline
\end{tabular}

\section{Invariância Métrica por Sexo}

A invariância foi testada para o modelo de segunda ordem proposto no presente trabalho. Para o modelo configural, as cargas fatoriais foram significativas para todos os itens e fatores para ambos os sexos. O modelo inicial obtido apresentou ajuste de $\chi^{2}(g l=2706)=3925,38$ $(p<0,001), \chi^{2} / g l=1,45$; CFI $=0,90$; TLI=0,89; RMSEA $=0,046(\mathrm{IC} 95 \%$ [0,043; 0,049]). O ajuste dos modelos separadamente para cada sexo resultou para o sexo masculino um $\chi^{2}=1777,01$ e sexo feminino, $\chi^{2}=2148,36$. A discrepância de ajuste para ambos os modelos é elevada, indicando a priori um ajuste mais adequado para a amostra masculina.

Considerando as restrições subsequentes, a invariância métrica (cargas fatoriais) não apresentou uma diferença significativa de ajuste para o teste de $\chi^{2}$ de diferença entre os grupos $\left(\Delta \chi^{2}(\Delta g l=50)=59,51\right.$; $p=0,168)$. Diferenças significativas foram detectadas para a invariância escalar $\left(\Delta \chi^{2}(\Delta g l=32)=56,55 ; p=0,004\right)$ e para a invariância das médias das variáveis latentes $\left(\Delta \chi^{2}(\Delta g l=22)=38,00 ; p=0,018\right)$. Por fim, a invariância dos resíduos apresentou o maior nível de discrepância 
$\left(\Delta \chi^{2}(\Delta g l=54)=85,82 ; p=0,004\right)$. Todos os três primeiros modelos mantiveram o mesmo grau de ajuste para o CFI robusto de 0,90 e RMSEA robusto de 0,046, indicando que as diferenças foram restritas ao teste de comparação para o $\chi^{2}$. Para as médias e resíduos, o CFI robusto reduz para 0,89 , mas o RMSEA se mantém com valor em 0,046 .

Tabela 2

Estimativas para os Fatores de Ordem Superior Incluindo Carga, Erro Padrão (EP) e p-Valor, Incluindo Correlação entre os Fatores de Segunda Ordem (Fim da Tabela)

\begin{tabular}{|c|c|c|c|c|c|c|c|}
\hline Fator & carga fatorial & $E P$ & $p$-valor & Fator & carga fatorial & $E P$ & $p$-valor \\
\hline $\mathrm{H} 1$ & & & & $\mathrm{H} 4$ & & & \\
\hline Realização & 0,77 & 0,10 & $<0,001$ & Solidão & 0,68 & 0,06 & $<0,001$ \\
\hline Sentido & 0,87 & 0,17 & $<0,001$ & Controle & 0,45 & 0,07 & $<0,001$ \\
\hline Otimismo & 0,85 & 0,15 & $<0,001$ & Negativos & 0,86 & 0,07 & $<0,001$ \\
\hline Satisfação & 0,88 & 0,17 & $<0,001$ & & & & \\
\hline Positivos & 0,83 & 0,12 & $<0,001$ & Fatores & Coef. & $E P$ & $p$-valor \\
\hline $\mathrm{H} 2$ & & & & $\mathrm{H} 4 \leftrightarrow$ & & & \\
\hline Flow & 0,78 & 0,14 & $<0,001$ & H3 & 0,39 & 0,07 & $<0,001$ \\
\hline Habilidades & 0,77 & 0,12 & $<0,001$ & $\mathrm{H} 2$ & 0,48 & 0,07 & $<0,001$ \\
\hline Aprendizado & 0,76 & 0,14 & $<0,001$ & $\mathrm{H} 1$ & 0,66 & 0,06 & $<0,001$ \\
\hline Eficácia & 0,82 & 0,17 & $<0,001$ & H3 ↔ & & & \\
\hline Autoestima & 0,88 & 0,21 & $<0,001$ & $\mathrm{H} 2$ & 0,67 & 0,05 & $<0,001$ \\
\hline H3 & & & & $\mathrm{H} 1$ & 0,58 & 0,05 & $<0,001$ \\
\hline Apoio & 0,62 & 0,12 & $<0,001$ & $\mathrm{H} 2 \leftrightarrow$ & & & \\
\hline Comunidade & 0,53 & 0,08 & $<0,001$ & $\mathrm{H} 1$ & 0,89 & 0,02 & $<0,001$ \\
\hline Confiança & 0,80 & 0,17 & $<0,001$ & - & - & - & - \\
\hline Respeito & 0,75 & 0,17 & $<0,001$ & - & - & - & - \\
\hline Pertencimento & 0,63 & 0,09 & $<0,001$ & - & - & - & - \\
\hline
\end{tabular}

\section{Consistência Interna}

Coeficientes de consistência interna foram obtidos para o conjunto de itens, considerando os fatores de primeira e segunda ordem. Para cada fator, foi estimado o coeficiente ômega de McDonald. Os resultados estão apresentados na Tabela 3 .

Tabela 3

Coeficiente de Consistência Alfa de Cronbach para Fatores de Primeira e Segunda Ordem

\begin{tabular}{|c|c|c|c|}
\hline Fator & Ômega & Fator & Ômega \\
\hline Apoio & 0,69 & Autoestima & 0,81 \\
\hline Comunidade & 0,71 & Controle & 0,75 \\
\hline Confiança & 0,76 & Sentido & 0,83 \\
\hline Respeito & 0,80 & Otimismo & 0,87 \\
\hline Solidão & 0,70 & Satisfação & 0,87 \\
\hline Pertencimento & 0,86 & Sentimentos positivos & 0,92 \\
\hline Flow & 0,75 & Sentimentos negativos & 0,88 \\
\hline Habilidades & 0,84 & $\mathrm{H} 1$ & 0,94 \\
\hline Aprendizado & 0,74 & $\mathrm{H} 2$ & 0,90 \\
\hline Realização & 0,88 & H3 & 0,85 \\
\hline Auto eficácia & 0,74 & $\mathrm{H} 4$ & 0,82 \\
\hline
\end{tabular}

O menor valor obtido foi para a dimensão Apoio, que apresentou um valor de $\omega=0,69$. O coeficiente médio foi de 0,80 para as dimensões de primeira ordem e 0,88 para as dimensões de segunda ordem. 


\section{Validade Convergente}

A validade convergente foi estimada dando continuidade ao framework de análises por modelagem de equações estruturais. No preparo das medidas, apenas o item 4 da medida de felicidade foi removido por apresentar carga fatorial inferior ao mínimo recomendado $(\lambda=0,28)$. O modelo obteve um ajuste de $\chi^{2}(g l=1982)=3458,65(p<0,001) ; \chi^{2} / g l=1,75 ; \mathrm{CFI}=0,90$; TLI $=0,90 ;$ RMSEA $=0,043(\mathrm{IC}=95 \%[0,040 ; 0,045]) . \mathrm{O}$ ajuste foi estimado como razoável para o modelo, considerando especialmente a complexidade com a inserção do modelo métrico de todas as medidas. Todos os coeficientes de regressão resultaram em significativos, sendo o menor entre $\mathrm{H} 4$ e florescimento $(\lambda=0,16 ; \mathrm{EP}=0,04$; $\mathrm{p}=0,034)$ e o maior entre H1 e felicidade $(\lambda=0,62$; $\mathrm{EP}=0,05 ; p \leq 0,001)$. A média dos caminhos e erros da variável felicidade para os fatores de IAP foi de $\lambda=0,46$ e $\mathrm{EP}=0,04$, enquanto para florescimento foi de $\lambda=0,32$ e $\mathrm{EP}=0,04$. Para os fatores de primeira ordem, o modelo atingiu um ajuste de $\chi^{2}(g l=1825)=3102,64(p<0,001)$, $\chi^{2} / g l=1,70 ; \quad$ CFI $=0,92 ; \quad$ TLI $=0,90 ; \quad$ RMSEA $=0,041$ $(\mathrm{IC}=95 \%[0,039 ; 0,044])$. Nesse cenário, parte dos fatores de primeira ordem não apresentou relação com as variáveis de referência, mas todas apresentaram pelo menos um coeficiente significativo. Com florescimento, três fatores não apresentaram coeficientes significativos, dentre eles: 1 . apoio $(\lambda=0,17 ; \mathrm{EP}=0,03 ; p=0,059), 2$. solidão $(\lambda=0,08 ; \mathrm{EP}=0,07 ; p=0,284)$ e 3 . controle $(\lambda=0,12$; $\mathrm{EP}=0,05 ; p=0,109)$. Para a medida de felicidade, apenas solidão apresentou um valor limítrofe $(\lambda=0,14$; $\mathrm{EP}=0,05 ; p=0,054)$.

\section{Discussão}

O Inventário Abrangente de Prosperidade (IAP) é uma medida com estrutura composta por 18 fatores de primeira ordem, que agregam um total de três itens por fator. Essa quantidade de itens por fator é considerada, na literatura, como o limite de itens inferior mínimo aceito para cada variável latente (Brown, 2015;; Hair, 2014). Portanto, essa estrutura representa um desafio computacional em função dos parâmetros do modelo, além de um desafio de replicabilidade, considerando a elevada complexidade do instrumento. Por outro lado, a proposta de medida desenvolvida por Su et al. (2014) tem como ponto forte a possibilidade de mapear um grande número de variáveis referentes à prosperidade, com indicadores ágeis.

A análise da medida, para uso no Brasil, deve considerar os aspectos relacionados à estrutura original proposta em primeira e segunda ordem, assim como a metodologia. Primeiramente, dentre os sete fatores de segunda ordem previstos, quatro apresentam apenas uma única variável latente de primeira ordem subordinada. Em termos metodológicos, a estrutura representa redundância métrica, considerando que a lógica de composição por hipótese reflexiva demanda múltiplos indicadores para sua justificação (Hair, 2014). Enquanto a regra é justificada em termos de estimativa apenas para a relação entre item e fator, os autores não apresentaram uma justificativa teórica para a composição dessas estruturas de segunda ordem, a não ser como grau equivalente aos demais fatores na mesma instância.

O presente estudo, no entanto, propôs uma combinação de estratégias de análise fatorial confirmatória e análise de redes, com o objetivo de identificar uma nova estrutura adequada à lógica de estimativa de variáveis latentes. Ainda que os nossos resultados indiquem que a nova estrutura não apresenta índices de ajuste estatisticamente distintos da originalmente proposta pelos autores, a nova estrutura apresenta um potencial de interpretação, além do fundamento teórico, imprescindível na metodologia de AFC.

Neste estudo, os fatores de primeira ordem se agruparam em quatro dimensões, a saber: Dimensão 1 - realização (F10), sentido (F14), otimismo (F15), satisfação (F16) e emoções positivas (F17), denominada satisfação com a vida; Dimensão 2 - flow (F7), habilidades (F8), aprendizado (F9), eficácia (F11) e autoestima (F12), denominada domínio do ambiente; Dimensão 3 - apoio (F1), comunidade (F2), confiança (F3), respeito (F4) e pertencimento $(\mathrm{F} 6)$, denominada relações interpessoais positivas; Dimensão 4 - solidão (F5), controle (F13) e emoções negativas (F18), denominada isolamento e negatividade.

As quatro dimensões de segunda ordem obtidas neste estudo podem ser justificadas com base nas raízes filosóficas das perspectivas hedônicas e eudaimônicas do bem-estar, assim como das teorias humanistas e fundamentos teóricos da Psicologia do Desenvolvimento (Dagenais-desmarais, Mendonça, Ferreira, \& Savoie, 2017). A dimensão Satisfação com vida engloba uma vida de sentido e propósito, perspectivas positivas em relação ao futuro e vivência de afetos positivos. A dimensão Domínio do ambiente abrange a crença numa vida próspera e nas habilidades para obter uma vida próspera. A dimensão denominada Relações interpessoais positivas envolve o relacionamento interpessoal com a comunidade, redes de apoio e sentimento de pertença social. A última dimensão denominada Isolamento e negatividade engloba uma vida solitária, a insegurança, falta de controle e de autonomia no ambiente, assim como a vivência de emoções negativas. Os itens dessa dimensão devem ser operacionalizados inversamente.

Por outro lado, as evidências da invariância métrica para sexo apontam para problemas potenciais. Em primeiro lugar, a medida aparentemente indica um ajuste mais adequado ao público masculino, porém o $\chi^{2} \mathrm{de}$ ajuste apresentou uma inflação significativa no caso do grupo feminino. A investigação da invariância métrica aponta que as cargas fatoriais podem ser equiparadas sem prejuízo da qualidade da medida, porém o mesmo não 
se repete para o caso dos demais aspectos de invariância - escalar, médias e restritas. Em estudos posteriores será essencial averiguar a especificidade ou invariância parcial considerando os itens, seja pelo próprio método de análise fatorial confirmatória ou ainda por funcionamento diferencial dos itens no paradigma de Teoria de Resposta ao Item (Ayala, 2009).

Ao examinar as demais propriedades do IAP, foram identificados índices satisfatórios de consistência interna, sendo que apenas a dimensão de primeira ordem Apoio apresentou valor limítrofe ao recomendado na literatura científica, porém ainda dentro de uma margem aceitável. Ao considerar as evidências de validade convergente com as variáveis de felicidade e florescimento, o nosso estudo também encontrou níveis que confirmam sua qualidade para o tipo de evidência em questão, confirmando os pressupostos de Su et al. (2014) e Duan et al. (2018).

Este estudo apresenta contribuições teóricas e metodológicas, especialmente para verificação da dimensionalidade ao incluir o método de redes, no emprego de análise mista. A preferência pelo uso desse método consistiu em suas evidências de estabilidade quando comparados aos demais métodos de confirmação de dimensionalidade (Golino \& Chirstensen, 2019). Além disso, o método de redes possibilitou encontrar soluções satisfatórias para a estrutura de segunda ordem. O presente trabalho também oferece uma contribuição singular para a comparação dos grupos, considerando o possível efeito da variável sexo. Por fim, o atual estudo não inclui apenas informações de aspectos estruturais da validade, mas também um critério externo à escala na forma das medidas de florescimento e felicidade.

Dentre as limitações, pode ser afirmado que ainda são necessárias evidências mais amplas, como a caracterização de uma rede nomológica de variáveis, incluindo outros critérios, além dos citados. Estudos futuros devem também investigar aspectos específicos da invariância parcial de cada uma das unidades estruturais do instrumento. Em que pese as limitações, o presente estudo deve ser considerado um passo inicial de conhecimento sobre as propriedades psicométricas da IAP no Brasil, sendo esperado que futuramente sejam feitas avaliações que incluam outros paradigmas métricos.

\section{Referências}

Ayala RJ. (2009). The theory and application of Item response theory. New York: Guilford Publishing.

Borsa, J. C., Damásio, B. F., \& Bandeira, D. R. (2012). Adaptação e validação de instrumentos psicológicos entre culturas: Algumas considerações. Paidéia, 22(53), 423-432. doi: 10.1590/s0103-863×2012000300014

Brown, T. A. (2015). Confirmatory factor analysis for applied research (Second edition). New York; London: The Guilford Press.

Chaaban, J., Irani, A., \& Khoury, A. (2015). The Composite Global Well-Being Index (CGWBI): A new multi-dimensional measure of human development. Social Indicators Research, 129(1), 465-487. doi:10.1007/s11205-015-1112-5

Csikszentmihalyi, M. (1996). Creativity: The work and lives of 91 eminent people. HarperCollins.

Dagenais-desmarais, V., Mendonça, H., Ferreira, M. C., \& Savoie, A. (2017). Psychological Well-Being at work: Where are we and where do we go from Here? Em A. M. Rossi, J. A. Meurs, \& P. L. Perrewé (Eds.). Stress and quality of Working Life - Conceptualizing and Assessing Stress (pp. 65-84). 1ed. Charlotte, NC: Information Age Publishing.

Deci, E. L., \& Ryan, R. M. (2008). Self-determination theory: A macrotheory of human motivation, development, and health. Canadian Psychology/Psychologie Canadienne, 49(3), 182-185. doi:10.1037/a0012801

Diener, E. (1984). Subjective well-being. Psychological Bulletin, 95(3), 542-575. doi:10.1037/0033-2909.95.3.542

Diener, E. (1999). Introduction to the special section on the structure of emotion. Journal of Personality and Social Psychology, 76(5), 803. doi: $10.1037 / 0022-3514.76 .5 .803$

Diener, E. (2006). Guidelines for National indicators of Subjective Well-Being an Ill-Being. Applied Research in Quality of Life, 1(2), 151-157. doi:10.1007/s11482-006-9007-x

Diener, E., Sandvik, E., \& Pavot, W. (2009). Happiness is the Frequency, Not the Intensity, of Positive Versus Negative Affect. Em E. Diener (Ed.), Social Indicators Research Series (pp. 213-231). Springer, Dordrecht. doi: 10.1007/978-90-481-2354-4_10

Diener, E., Ng, W., Harter, J., \& Arora, R. (2010). Wealth and happiness across the world: Material prosperity predicts life evaluation, whereas psychosocial prosperity predicts positive feeling. Journal of Personality and Social Psychology, 99(1), 52-61. doi: 10.1037/a0018066

Duan, W., \& Xie, D. (2016). Measuring adolescent flourishing: Psychometric Properties of Flourishing Scale in a Sample of Chinese Adolescents. Journal of Psychoeducational Assessment, 37(1), 131-135. doi:10.1177/0734282916655504

Duan, W., Fei, Y., Zhao, J., \& Guo, X. (2018). Incremental validity of the comprehensive inventory of thriving in predicting self-reporting mental and physical health among community populations. Journal of Health Psychology, 5, 1-8. doi: 10.1177/1359105318755265

Duan, W., Guan, Y., \& Gan, F. (2016). Brief Inventory of Thriving. Chinese Sociological Dialogue, 1(1), 15-31. doi:10.1177/2397200916665230

Golino, H., \& Christensen, A. (2019). EGAnet: Exploratory Graph Analysis: A Framework for Estimating the Number of Dimensions in Multivariate Data Using Network Psychometrics. RCRAN.

Hair, J. F. (2014). Multivariate data analysis (7. ed., Pearson new internat. ed). Harlow: Pearson.

Hildenbrand, K., Sacramento, C. A., \& Binnewies, C. (2018). Transformational leadership and burnout: The role of thriving and followers' openness to experience. Journal of Occupational Health Psychology, 23(1), 31-43. doi: 10.1037/ocp0000051

Huppert, F. A., \& So, T. T. C. (2013). Flourishing across Europe: Application of a new conceptual framework for defining well-being. Social Indicators Research, 110(3), 837-861. doi: 10.1007/s11205-011-9966-7 
International Test Commission. (2010). International Test Commission guidelines for translating and adapting tests. Recuperado de http://www. intestcom.org/upload/sitefi les/40.pdf

Jorgensen, T. (2018). SemTools: Useful tools for structural equation modeling. RCRAN.

Joshanloo, M., Sirgy, M. J., \& Park, J. (2018). Directionality of the relationship between social well-being and subjective well-being: Evidence from a 20-year longitudinal study. Quality of Life Research, 27(8), 2137-2145. doi: 10.1007/s11136-018-1865-9

Lucas, R. E., Dyrenforth, P. S., \& Diener, E. (2008). Four Myths about Subjective Well-being. Social and Personality Psychology Compass, 2(5), 2001-2015. doi: 10.1111/j.1751-9004.2008.00140.x

Lyubomirsky, S., \& Lepper, H. S. (1999). A measure of subjective happiness: Preliminary reliability and construct validation. Social indicators Research, 46(2), 137-155. doi: 10.1023/A:1006824100041

McGregor, I., \& Little, B. R. (1998). Personal projects, happiness, and meaning: On doing well and being yourself. Journal of Personality and Social Psychology, 74(2), 494-512. doi: 10.1037/0022-3514.74.2.494

Mendonça, H., \& Ferreira, M. C. (2018) Florescimento no trabalho (flourishing): Novo conceito, velho fenômeno. Em A. C. S. Vazquez, \& C. S. Hutz (Org.). Aplicações da psicologia positiva: Trabalho e organizações (pp. 187-199). $1^{\mathrm{a}}$ ed. São Paulo: Hogrefe CETEPP.

Mendonça, H., Caetano, A., Ferreira, M. C., Sousa, I. F., \& Silva, A. J. (2014). Florescimento no trabalho. Em M. M. M. Siqueira (Org.). Novas medidas do comportamento organizacional: Ferramentas de diagnóstico e de gestão (pp. 172-177) $1^{\mathrm{a}}$ ed. Porto Alegre: Artmed Editora Ltda.

R Core Team. (2018). R: A language and environment for statistical computing. R Foundation for Statistical Computing, Viena, Áustria.

Reichardt, J., \& Bornholdt, S. (2006). Statistical mechanics of community detection. Physical Review E, 74(1). doi:10.1103/physreve.74.016110

Revelle, W. (2018). Psych: procedures for personality and psychological research. Northwestern University, Evanston, Illinois, USA.

Revelle, W., \& Zinbarg, R. E. (2009). Coefficients Alpha, Beta, Omega, and the glb: Comments on Sijtsma. Psychometrika, 74(1), 145-154. doi: 10.1007/s11336-008-9102-z

Rosseel, Y. (2018). Package 'Lavaan'. RCRAN.

Ryan, R. M., \& Deci, E. L. (2000). Intrinsic and extrinsic motivations: Classic definitions and new directions. Contemporary Educational Psychology, 25(1), 54-67. doi: 10.1006/ceps.1999.1020

Ryff, C. D. (1989). Happiness is everything, or is it? Explorations on the meaning of psychological well-being. Journal of Personality and Social Psychology, 57(6), 1069-1081. doi: 10.1037/0022-3514.57.6.1069

Seligman, M. (2018). PERMA and the building blocks of well-being. The Journal of Positive Psychology, 13(4), 333-335. doi: $10.1080 / 17439760.2018 .1437466$

Spagnoli, P., Caetano, A., \& Silva, A. (2010). Psychometric Properties of a Portuguese Version of the Subjective Happiness Scale. Social Indicators Research, 105(1), 137-143. doi: 10.1007/s11205-010-9769-2

Su, R., Tay, L., \& Diener, E. (2014). The Development and Validation of the Comprehensive Inventory of Thriving (CIT) and the Brief Inventory of Thriving (BIT). Applied Psychology: Health and Well-Being, 6(3), 251-279. doi: 10.1111/aphw.12027

Tang, X., Duan, W., Wang, Z., \& Liu, T. (2016). Psychometric Evaluation of the Simplified Chinese Version of Flourishing Scale. Research on Social Work Practice, 26(5), 591-599. doi: 10.1177/1049731514557832

Tong, K. K., \& Wang, Y. Y. (2017). Validation of the flourishing scale and scale of positive and negative experience in a Chinese community sample. PLOS ONE, 12(8), e0181616. doi: 10.1371/journal.pone.0181616

Wiese, C. W., Tay, L., Su, R., \& Diener, E. (2018). Measuring thriving across nations: Examining the Measurement Equivalence of the Comprehensive Inventory of Thriving (CIT) and the Brief Inventory of Thriving (BIT). Applied Psychology: Health and Well-Being, 10(1), 127-148. doi: 10.1111/aphw.12119

Wrosch, C., Jobin, J., \& Scheier, M. F. (2016). Do the Emotional Benefits of Optimism Vary Across Older Adulthood? A Life Span Perspective. Journal of Personality, 85(3), 388-397. doi: 10.1111/jopy.12247

\section{Sobre os autores}

Helenides Mendonça é psicóloga, doutora em Psicologia pela UnB, com pós-doutorado no Instituto Universitário de Lisboa - ISCT/ IUL. Professora titular da Pontifica Universidade Católica de Goiás (PUC-GO).

Pedro P. Pires é psicólogo, doutor em psicologia pela Universidade Federal do Rio de Janeiro com PDSE pela University of Maryland. Professor ajunto do departamento de psicometria e do programa de pós-graduação em psicologia (stricto sensu) da UFRJ.

Luciana Pacheco é odontóloga, graduanda de psicologia pela da Pontifica Universidade Católica de Goiás (PUC-GO), na linha de pesquisa Cultura e Saúde.

Ariana Fidelis é psicóloga, doutoranda pela Pontifica Universidade Católica de Goiás (PUC-GO). Professora horista da graduação da PUC-GO.

Isabella de M. Ramos Caiado é médica (Centro Universitário Barão de Mauá). Atua no programa de saúde da família do município de Aparecida de Goiânia, onde desenvolve pesquisas na área de bem-estar. 